UCRL-ID-131699

\title{
Enhanced Ultrasonic Characterization of Assemblies, TLL_19
}

\author{
Graham Thomas \& Diane Chinn \\ Lawrence Livermore National Laboratory \\ Livermore, CA 94550
}

September, 1998

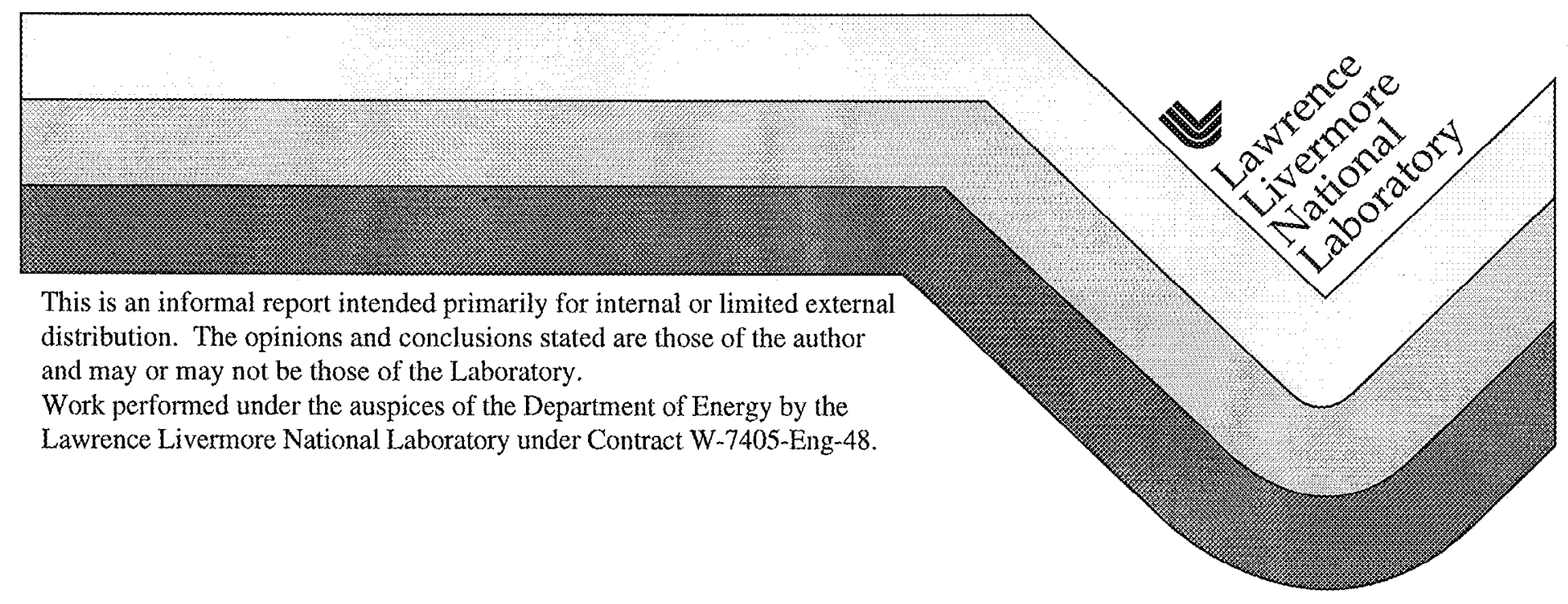




\section{DISCLAIMER}

This document was prepared as an account of work sponsored by an agency of the United States Government. Neither the United States Government nor the University of California nor any of their employees, makes any warranty, express or implied, or assumes any legal liability or responsibility for the accuracy, completeness, or usefulness of any information, apparatus, product, or process disclosed, or represents that its use would not infringe privately owned rights. Reference herein to any specific commercial product, process, or service by trade name, trademark, manufacturer, or otherwise, does not necessarily constitute or imply its endorsement, recommendation, or favoring by the United States Government or the University of California. The views and opinions of authors expressed herein do not necessarily state or reflect those of the United States Government or the University of California, and shall not be used for advertising or product endorsement purposes.

This report has been reproduced directly from the best available copy.

Available to DOE and DOE contractors from the Office of Scientific and Technical Information

P.O. Box 62, Oak Ridge, TN 37831

Prices available from (615) 576-8401, FTS 626-8401

Available to the public from the

National Technical Information Service

U.S. Department of Commerce

5285 Port Royal Rd.,

Springfield, VA 22161 


\title{
Enhanced Ultrasonic Characterization of Assemblies, TLL_19
}

\section{Graham Thomas and Diane Chinn}

\begin{abstract}
Bonded joints, such as the autoclave bond, are critical to the performance of weapon systems. A nondestructive method to assess the integrity of these bonds is needed to certify the weapon for extended life. This project is developing ultrasonic technologies for bond quality assessment. Existing ultrasonic technology easily maps totally unbonded areas in a bond line but does not measure the quality of the bond. We are extracting information from the ultrasonic signals to quantify the mechanical properties and assess the durability of the bond. Our approach is based on advanced signal processing and artificial intelligence techniques that process information from the ultrasonic signal after it interacts with the bondline. Computer algorithms recognize variations in bond quality from the acoustic signals. The ultrasonic signal processing and bond classification software will be installed on ultrasonic scanners at the appropriate sites.
\end{abstract}

\section{Objectives}

This project is developing ultrasonic nondestructive evaluation technology for assessing solid state bonds. Specifically, we are expanding our ultrasonic signal processing and defect characterization technology to interrogate bondlines and determine their quality. Since bonds degrade with time, we will be able to track the aging and predict the remaining life. This nondestructive evaluation technology will assess the quality of autoclave bonds as well as other solid state and adhesive bonds. Lawrence Livermore National Laboratory is working closely with team members from Y-12, Allied Signal Kansas City, and Savannah River Site to implement ultrasonic data acquisition, bond classification, and display techniques. We will implement the advanced algorithms developed by this project on a computer controlled scanning system. The scanning system will implement the ultrasonic bond quality determination procedures on weapon components. Ultimately, each partner will implement the technology developed by this project into their ultrasonic systems. 
collaborating with another project to develop an automated, high resolution ultrasonic scanning system for pit inspection.

A program within the Nuclear Weapons Complex has been organized with the main participants (LLNL, Allied Signal, Y-12, and SRS). Interest in this project has been expressed by LANL and AWE. We are formulating a focus exchange with AWE based on this project.

We enlarged our set of surrogate bond samples and implemented our signal processing software to demonstrate feasibility. Table 1 shows the matrix of diffusion bond specimens which represent various bond quality. Samples are made by diffusion bonding aluminum to copper. Some of the aluminum and copper interfaces are prepared by depositing a 5 micrometer thick copper or zinc interlayer before bonding as indicated in Table 1. Diffusion bonding occurs at $500^{\circ} \mathrm{C}$ or $550{ }^{\circ} \mathrm{C}$ at 175 psi pressure. Bonding time is either 60 minutes or 90 minutes.

We acquired ultrasonic signals from the bond and processed the signals with our algorithms. Figure 1 illustrates our protocol for developing bond quality determination algorithms. To date we have completed the data acquisition, signal processing, and feature extract phases of the bond classification protocol on a set of samples. Figure 2 displays the results of this phase. Total reflected energy is one feature which indicates bond quality. Fig. 2 shows images mapping reflected energy from the bond interface over a $20 \mathrm{~cm} \times 20$ $\mathrm{cm}$ area of the bond in five of the samples. All images are shown scaled to the same color scale. Total energy [volts ${ }^{2}$ ] in the $20 \mathrm{~cm} \times 20 \mathrm{~cm}$ region of interest is noted. Variations in bond quality are evident both within a bond and from bond to bond. This data demonstrates the feasibility of distinguishing between differing bond qualities.

A suite of signal characteristics (features) will be extracted and the better features will be processed with classification software for predicting bond quality. Figure 2 deals with a single feature, total energy, whereas more features will improve the performance of the classification algorithm. Many more bond specimens are needed to build confidence in the algorithms predictive ability.

To implement the bond quality measuring technology, we need a system to acquire ultrasonic data from the entire bonded interface. Such an ultrasonic scanner is being 


\section{Milestones}

We accomplished the scheduled milestones as described in the following text.

A complex wide program was organized with the main participants (LLNL, Allied Signal, Y-12, and Savannah River Site). Also interest in this project has been expressed by Los Alamos National Laboratory and the Atomic Weapons Establishment (U. K.). We are in the process of formulating a focus exchange with the Atomic Weapons Establishment based on this project. The team chose standardized hardware and software needed for the project based on LLNL's experience with the specialized type of hardware and software required. Each site acquired the necessary ultrasonic instrumentation and data acquisition systems. We identified sources of surrogate and real bond specimens and mechanisms to acquire ultrasonic data from these bonds. Los Alamos National Laboratory and the Atomic Weapons Establishment will supply ultrasonic bond data from real parts and share in the resulting classification algorithms. Ultrasonic data from well-characterized specimens are an extremely important part of this project. A large set of realistic bond data will facilitate a robust classification algorithm.

Signal processing algorithms were written to process the acoustic data and reject noise. Feature extraction and feature selection software have been written to identify the pertinent characteristics of the ultrasonic waveforms for bond quality determination. We are developing classification software based on statistical pattern recognition and probabilistic neural networks. Figure 1 illustrates our protocol for developing a bond quality classification algorithm.

We implemented classification techniques to correlate information contained in ultrasonic bondline signals with the quality of the bond. We fabricated a set of surrogate bond samples and implemented our signal processing software to demonstrate feasibility. Table 1 shows the variety of diffusion bond specimens that represent different bond qualities. To determine if any undesirable debonds were present, we ultrasonically imaged the diffusion bond interfaces using the pulse-echo technique. Unbonded samples are easy to detect and are not the goal of this project, therefore all unbonds were noted and those specimens were ignored.

To implement the bond quality measuring technology, we need a system to acquire ultrasonic data from the entire interface. Such an ultrasonic scanner is being developed. The scanning equipment will be enclosed in a glove box and the electronics will remain 
outside of the contaminated area. We designed and are currently fabricating an automated ultrasonic scanning system for adhesive bonds. This system, which was funded by another program, provides high resolution ultrasonic data acquisition for parts with a similar geometry to those of interest for this program. We will build a dedicated system based on the adhesive bond scanner for this program. Ultimately the ultrasonic scanner will run the classification algorithms being developed by this project and will process the ultrasonic signals to render an image of bond quality.

\section{Deliverables}

- Meetings were held to organize this project so that each participant has been assigned deliverables. These deliverables will combine to benefit all. We are also working with sites that are not funded under this project but who are very interested in the technology and have volunteered to support the effort with data and knowledge.

- Surrogate bond samples with programmed bond qualities have been manufactured.

- Ultrasonic data was acquired on the surrogate samples.

- Software to extract features from ultrasonic signals and classify bond strength was written.

- A dedicated ultrasonic scanning and data acquisition system has been designed for evaluating autoclave bonds.

\section{Accomplishments}

We developed a procedure to produce bond quality classification algorithms. These algorithms extract information contained in the ultrasonic signal reflected by the bonded interface. The first step was identifying and writing signal processing algorithms to process the acoustic data and to reject noise. Next we wrote feature extraction and feature selection software to recognize the pertinent characteristics of the ultrasonic waveforms for bond quality determination. We then developed the classification software based on statistical pattern recognition and probabilistic neural networks. We began implementing classification techniques to correlate information contained in ultrasonic bondline signals with the quality of the bond. We fabricated a set of surrogate bond samples and implemented our signal processing software to demonstrate feasibility. Lastly, we are 
developed. The scanning equipment will be enclosed in a glove box and the electronics will remain outside of the contaminated area.

We designed and are fabricating an automated ultrasonic scanning system for adhesive bonds. This system will provide high resolution ultrasonic data acquisition for real parts. Ultimately this ultrasonic scanner running the classification algorithms being developed under this project will process the ultrasonic signals and render an image of bond quality.

We had an opportunity to demonstrate the feasibility of ultrasonically imaging a diffusion bond surrogate specimen that had the exact dimensions of the autoclaved part. This sample was a small round core sample that is tensile tested as part of core surveillance. Figure 3 shows an ultrasonic image of the diffusion bond. The different colored areas in the image show variations in the bond. The feasibility of producing high resolution ultrasonic images was demonstrated.

\section{Summary}

We are working on ultrasonic technology to determine diffusion bond quality. After an ultrasonic signal interacts with a bond, it contains information about the quality of the interface. Signal processing algorithms extract information contained in the ultrasonic signal reflected by the bonded interface. The first step was identifying and writing algorithms to process the acoustic data and to reject noise. Next we wrote feature extraction and feature selection software to recognize the pertinent characteristics of the ultrasonic waveforms for bond quality determination. We then developed the classification software based on statistical pattern recognition and probabilistic neural networks. We began implementing classification techniques to correlate information contained in ultrasonic bondline signals with the quality of the bond. We fabricated a set of surrogate bond samples and implemented our signal processing software to demonstrate feasibility. Lastly, we are collaborating with another project to develop an automated, high resolution ultrasonic scanning system for pit inspection. We will implement the advanced algorithms developed by the project on this computer controlled system. The goal of this project is to implement the ultrasonic bond quality determination procedures on weapon components. Ultimately, each of the partners will implement the technology developed by this project into their ultrasonic systems. 


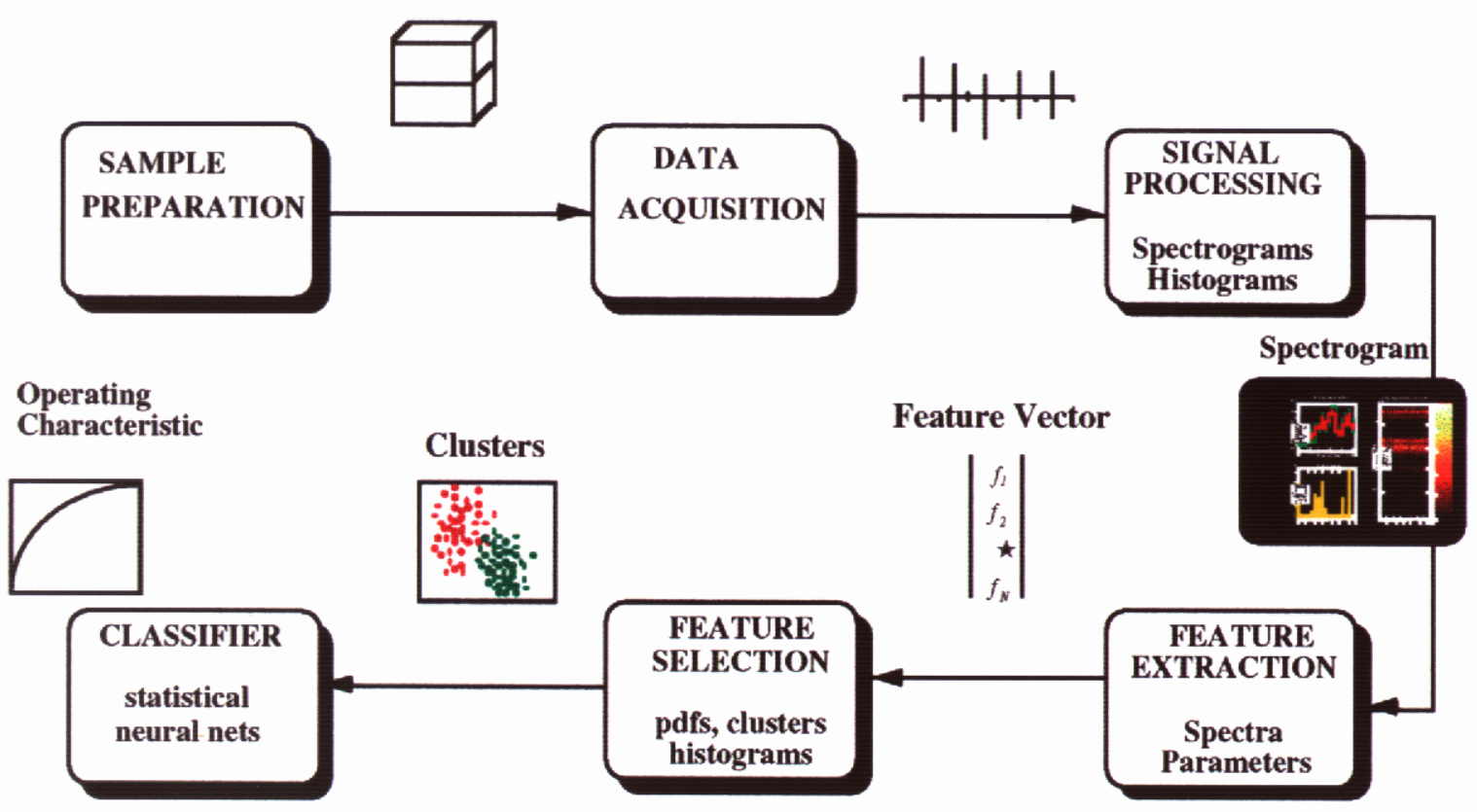

Fig. 1. The protocol for developing a bond quality classification algorithm is shown in this block diagram. 
Diffusion Bonding of $\mathrm{Al}$ to $\mathrm{Cu}$

\begin{tabular}{|c|c|c|c|c|c|c|}
\hline \multicolumn{5}{|c|}{ Surface Condtions } & \multicolumn{3}{|c|}{ Bonding Conditions, Plan/Actual } \\
& $\mathrm{Cu}$ & Al Sp\# & Cu Sp\# & Temp., C & $\begin{array}{c}\text { Bonding } \\
\text { Time, min. }\end{array}$ & $\begin{array}{c}\text { Bond } \\
\text { Pressure, } \\
\text { psi }\end{array}$ \\
\hline None & None & AlP2 & CuP1 & $550 / 535^{*}$ & 60 & 175 \\
\hline None & None & AlP1 & CuP2 & $550 / 535^{*}$ & 90 & 175 \\
\hline $\mathrm{Zn}$ & $\mathrm{Zn}$ & AlZn3 & CuZn2 & 500 & 60 & 175 \\
\hline $\mathrm{Zn}$ & $\mathrm{Zn}$ & AlZn1 & CuZn1 & 500 & 90 & 175 \\
\hline $\mathrm{Cu}$ & $\mathrm{Zn}$ & AlCu2 & CuZn3 & $550 / 535^{*}$ & 90 & 175 \\
\hline $\mathrm{Cu}$ & $\mathrm{Zn}$ & AlCu5 & CuZn4 & 500 & 90 & 175 \\
\hline $\mathrm{Zn}$ & None & AlZn4 & Cu2 & 500 & 60 & 175 \\
\hline $\mathrm{Zn}$ & None & AlZn2 & Cu4 & 500 & 90 & 175 \\
\hline $\mathrm{Cu}$ & None & AlCu4 & Cu5 & 550 & 60 & 175 \\
\hline $\mathrm{Cu}$ & None & AlCu1 & Cu1 & 550 & 90 & 175 \\
\hline
\end{tabular}

Table 1. A matrix of aluminum-copper bond samples allows us to study variations in bonds due to different surface preparations, bonding temperatures and bonding times. 


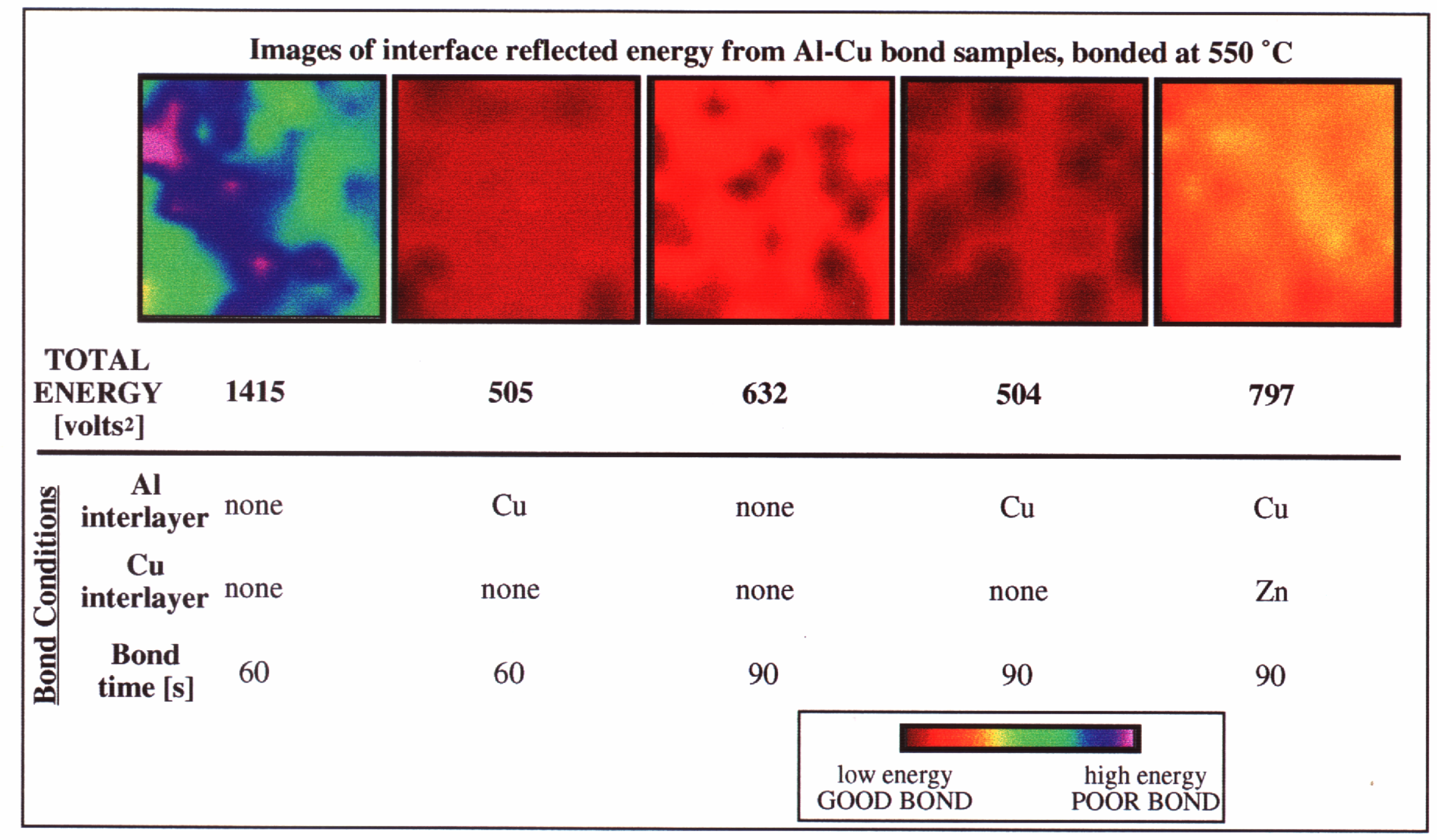

Fig. 2. Energy in the reflected wave from an $\mathrm{Al}-\mathrm{Cu}$ bond changes with bonding conditions. Images of a $20 \times 20 \mathrm{~mm}$ area of the interface show variations in the bond. At a bonding temperature of $550{ }^{\circ} \mathrm{C}$, the composition of the interlayer and the bond time affect bond quality. 


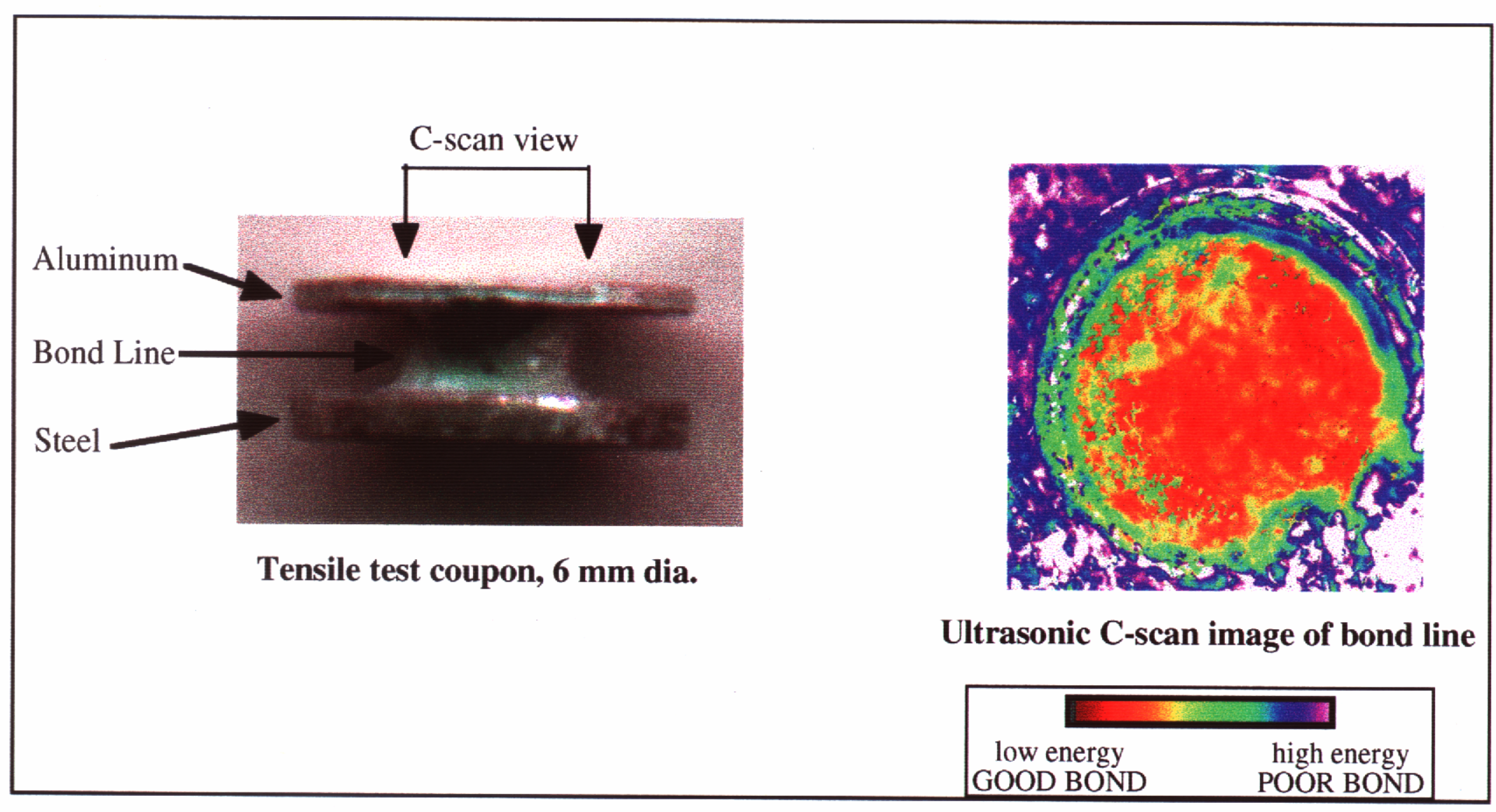

Fig. 3. An ultrasonic image of the diffusion bond on a surrogate tensile test coupon shows variations in bond quality. The image is produced by scanning an ultrasonic transducer normal to the aluminum side of the specimen and recording reflections from the bond line interface. 